\title{
A finite temperature bridging domain method for MD-FE coupling and application to a contact problem
}

\author{
G. Anciaux ${ }^{\mathrm{a}}$, S.B. Ramisetti ${ }^{\mathrm{a}}$, J.F. Molinari ${ }^{\mathrm{a}, *}$ \\ ${ }^{a}$ Ecole Polytechnique Fédérale de Lausanne (EPFL) \\ Faculté ENAC-IIS, Laboratoire de Simulation en Mécanique des Solides (http://lsms.epfl.ch/) \\ CH-1015 Lausanne, Switzerland
}

\begin{abstract}
A direct multiscale method coupling Molecular Dynamics to Finite Element simulations is introduced to study the contact area evolution of rough surfaces under normal loading. First, a description of the difficulties due to using the Bridging Domain Method at finite temperatures is discussed. This approach, which works well at low temperatures, is based on a projection, in an overlap region, of the atomic degrees of freedom on the coarser continuum description. It is shown that this leads to the emergence of a strong temperature gradient in the bridging zone. This has motivated the development of a simpler approach suitable for quasi-static contact problems conducted at constant but finite temperatures. This new approach is then applied to the normal loading of rough surfaces, in which the evolution of the real contact area with load is monitored. Surprisingly, the results show little influence of the contact area on temperature. However, the plastic events, in form of atomic reshuffling at the surface and dislocation activity, do clearly depend on temperature. The results show also a strong and temperature-dependent relaxation of the initial rough surfaces. This natural mechanism which alters atomic asperities brings to question the classical atomic description of roughness.
\end{abstract}

\section{Introduction}

Contact between surfaces is essential in many natural processes and technological applications. Nonetheless, our understanding of contact mechanics and its important by-products which are friction and wear can arguably still be considered at its infancy. In contact problems, experimental, numerical or theoretical analysis is complicated by the wide range of phenomena that occurs concurrently at various scales. In the last decades, the advancement of Atomic Force Microscopy has generated a breadth of fundamental experiments that give us new hopes of understanding the origins of frictional forces. The push towards nanoscience and nanotechnologies, which emphasize surface mechanisms, has only exacerbated this strong interest. Not surprisingly, the number of both continuum and atomic scale studies of contact has seen a strong and recent increase.

Perhaps one of the most fundamental sub problem of contact mechanics is that of two rough surfaces brought in normal loading. Traditional theories [1, 2, 3, 4] often assume that a stressed

${ }^{*}$ Corresponding author

Preprint submitted to Elsevier

March 11, 2014 
solid with a rough surface responds elastically to external load. This leads to a linear dependence of the contact area with the applied pressure. The idea is that the applied stress allows more and more asperities to contact and thus sustain load. The fractal nature of surfaces is often a key point to exploit statistical properties on deforming asperities leading to the described linear behavior. Numerical studies $[5,6,7,8,9]$ at both continuum and molecular levels showed that this trend is effective even when including plastic deformation. Finite element (FE) simulations [6] have also shown that the real contact is constituted of small clusters at which atomic interactions should dominate. This has motivated recent nanoscale contact simulations, in which the roughness dependency as well as the elastic-plastic contribution to the overall deformation were investigated $[10,11]$. However, few studies have analyzed the influence of temperature on surface roughness and contact evolution.

This is surprising since contact, especially during sliding motion, generates important temperature gradients due to plastic flow [12]. In this context surface topologies can be altered due to thermal expansion and the accompanying pressure confinement. Furthermore, heat generation and heat transfer through contacting asperities depend on the contacting profile [13]. The interdependency between surfaces and thermal conduction must be modelled correctly. Be it at the macroscale, for sliding or rolling contact, or at the nanoscale during nano-indentation or nanoscratching experiments, understanding temperature evolution and its influence on deformation mechanisms at interacting asperities is of crucial importance.

In this work, we focus on the effect of temperature on normal contact. The study is conducted at the nanoscale using molecular dynamics as the principal model. This necessitates long simulation runs to let temperature equilibrate. In order to reduce the computational costs, we introduce a direct multiscale coupling between molecular dynamics and a finite element engine.

One can distinguish two main coupling strategies. The first one merges interface atoms with boundary mesh nodes at a given interface in order to allow information passing $[14,15,16]$. Finite temperatures can be achieved by means of time averaging [17] or with an implicit modeling of thermal properties [18]. Nevertheless, the mesh constraint is very demanding. Indeed the mesh preparation needs the coupled nodes to match atomic sites. Furthermore, the mesh should then refine down to the atomic scale, which induces a large computational time.

The other family includes a bridging zone [19, 20,21, 22], which is a transition region where the atomic contribution is matched to continuum at a scale above. At low temperatures these methods were extensively studied to prevent spurious effects such as wave reflections [23]. To the best of our knowledge, finite temperature above hundreds of Kelvin were not considered in previous studies. In this paper, we will test the limitations of the Bridging Domain method, which applies a projection, before proposing a coupling strategy that can work well in threedimensional cases within a thermostated environment.

In the next section, we present the details about the Molecular Dynamics (MD) model used to represent a perfect crystal copper. Thermal expansion is taken into account so that zero internal pressure is maintained. Then we present the coupling methodology, starting with a demonstration of the inherent limitations of projective methods, and our solution to palliate these difficulties. In the fourth section we build a body with a free rough surface that is to be pressed against a rigid flat plate. Then, we report our results regarding the influence of temperature on contact and pressure measurements, as well as on plastic activity at the contacting interface. Finally a discussion about the stability of the constructed surfaces is presented before concluding. 


\section{MD model}

The purpose of this section is to describe the MD simulation model. The simulation model uses a perfect FCC copper crystal. The Cartesian reference frame of axes is set along the lattice directions. The Embedded Atom Method (EAM) [24] is used to model the inter-atomic interactions. The basic equations of the EAM potential are described as:

$$
\begin{aligned}
E_{t o t} & =\frac{1}{2} \sum_{i, j \neq i} V\left(r_{i j}\right)+\sum_{i} F\left(\rho_{i}\right) \\
\rho_{i} & =\sum_{j \neq i} \phi\left(r_{i j}\right)
\end{aligned}
$$

where $V$ is a pair potential, $\phi(r)$ is the electronic density contribution with respect to the distance $r$ of a neighbor atom, $\rho_{i}$ is the electronic density associated with atom $i, \mathbf{r}_{i}$ is the position of atom $i, \mathbf{r}_{i j}=\mathbf{r}_{j}-\mathbf{r}_{i}$ and $r_{i j}$ is the norm of the vector $\mathbf{r}_{i j}$. Then the force that is to be applied on a given atom $k$ is:

$$
\frac{\partial E_{t o t}}{\partial \mathbf{r}_{k}}=\sum_{i \neq k}\left\{V^{\prime}\left(r_{i k}\right)+\left[F^{\prime}\left(\rho_{i}\right)+F^{\prime}\left(\rho_{k}\right)\right] \phi^{\prime}\left(r_{i k}\right)\right\} \frac{\mathbf{r}_{i k}}{r_{i k}}
$$

EAM potentials commonly define pair potential and electronic density by means of tabulated functions. In our simulations we used the functions developed by Mishin [25] that accounts well for the dislocations activation energies. Also a time step of $1 \mathrm{fs}$ is used to integrate the governing equations of motion.

One difficulty in finite temperature MD studies is that the crystal lattice parameter (referred to " $a$ " later on) is temperature dependent. Thermal expansion must be taken into account in order to maintain constant pressure. We computed these lattice constants with a cubic simulation box made of 4000 copper atoms using NPT (constant number of atoms, pressure and temperature) ensemble. In these simulations the global domain size is allowed to vary in order to accommodate pressure and temperature effects. We introduced a zero pressure constraint and several temperatures were elected. Table 1 presents the obtained lattice constants as well as the thermal expansion coefficients. They match the values revealed by other studies [25]. The crystalline bodies used in our work were all built accounting for the thermal expansion.

\begin{tabular}{|c|c|c|}
\hline Temperature in Kelvin & Lattice constant in $\AA$ & Thermal expansion in $10^{-5} \mathrm{~K}^{-1}$ \\
\hline 100 & 3.620 & 1.518 \\
200 & 3.625 & 1.533 \\
300 & 3.631 & 1.552 \\
400 & 3.637 & 1.589 \\
500 & 3.643 & 1.605 \\
600 & 3.650 & 1.631 \\
700 & 3.657 & 1.666 \\
800 & 3.664 & 1.698 \\
900 & 3.671 & 1.731 \\
\hline
\end{tabular}

Table 1: Lattice constant and thermal expansion at different temperatures 


\section{Multiscale approach}

As mentioned earlier, we use a concurrent multiscale approach with atomic to continuum coupling to simulate the behavior of materials in contact. Full atomic details are retained in critical regions of the material where asperities are contacting (and exhibit a complex deformation behavior), while macro or continuum models are employed to describe deformation in regions more remote from the contact. Let us define $\Omega^{A}$ and $\Omega^{F E}$ the atomic domain and the continuum domain respectively. In $\Omega^{A}$ the model introduced in the previous section is used. In $\Omega^{F E}$ the well-known linear momentum balance expression for a continuum with no body force is used:

$$
\nabla \cdot \underline{\underline{\sigma}}=\rho \dot{\mathbf{v}} \quad \text { with } \quad \underline{\underline{\sigma}}=C: \underline{\underline{\epsilon}}
$$

where $\sigma$ is the Cauchy stress tensor, $\rho$ is the mass density, $\mathbf{v}$ is the velocity field and $C$ is the elasticity tensor. For the copper material we use a constitutive anisotropic law that has the following elasticity tensor:

$$
C=\left(\begin{array}{cccccc}
C_{11} & C_{12} & C_{12} & 0 & 0 & 0 \\
C_{12} & C_{11} & C_{12} & 0 & 0 & 0 \\
C_{12} & C_{12} & C_{11} & 0 & 0 & 0 \\
0 & 0 & 0 & C_{44} & 0 & 0 \\
0 & 0 & 0 & 0 & C_{44} & 0 \\
0 & 0 & 0 & 0 & 0 & C_{44}
\end{array}\right)
$$

The elastic constants $C_{11}, C_{12}$ and $C_{44}$ were computed in [26] at various temperatures and are recalled in table 2 .

\begin{tabular}{|c|c|c|c|}
\hline Temperature in Kelvin & $C_{11}$ & $C_{12}$ & $C_{44}$ \\
\hline 100 & 171.0 & 119.6 & 78.5 \\
200 & 163.2 & 114.8 & 75.4 \\
300 & 160.2 & 112.3 & 72.2 \\
400 & 152.1 & 109.2 & 69.9 \\
500 & 143.2 & 102.2 & 66.4 \\
600 & 140.4 & 99.8 & 61.6 \\
700 & 131.5 & 96.6 & 58.8 \\
800 & 123.8 & 94.4 & 54.8 \\
900 & 111.5 & 84.2 & 54.1 \\
1000 & 106.1 & 81.9 & 51.1 \\
\hline
\end{tabular}

Table 2: Elastic constants in GPa at different temperatures from [26]

In the Bridging Domain method [19, 20], FE and MD models are used to represent continuum and fine (atomic) scales respectively while a transition region allows the proper handling of wave reflections at low temperature [23]. But, to the best of our knowledge this method was never tested in a true finite temperature situation. In the following subsection we first recall the coupling details before presenting the problems occurring for finite temperatures. Ultimately we introduce a correction that prevents problems related to thermal fields. 


\subsection{Bridging Domain method}

We refer to the coupling method introduced by Belytschko and Xiao [19], which couples continuum models with molecular models using an overlapping region $\Omega^{R}$, in which both the continuum and molecular domains coexist. A global Hamiltonian is taken as the sum of the weighted Hamiltonians of both the molecular and continuum parts. The global multiscale Hamiltonian is simply given as

$$
H=E^{A}+E^{C}
$$

where $E^{A}$ and $E^{C}$ are the atomic and continuum Hamiltonian contributions, expressed as

$$
\begin{aligned}
& E^{A}=\sum_{\mathbf{q}_{\mathbf{i}} \in \Omega^{A} \backslash \Omega^{R}} E_{i}\left(\mathbf{q}_{\Omega^{A}}\right)+\sum_{q_{i} \in \Omega^{R}}\left(1-\alpha\left(\mathbf{X}_{\mathbf{i}}\right)\right) E_{i}\left(\mathbf{q}_{\Omega^{R}}\right) \\
& E^{C}=\sum_{e \in \Omega^{F E} \backslash \Omega^{R}} E_{e}\left(\mathbf{u}_{\Omega^{\mathrm{FE}}}\right)+\sum_{e \in \Omega^{R}} \int_{e} \alpha(\mathbf{X}) \delta E_{e}\left(\mathbf{u}_{\Omega^{R}}\right) d \mathbf{X}
\end{aligned}
$$

with $E_{i}$ the energy fraction for atom $i, \mathbf{X}_{i}$ its initial position, $E_{e}$ the energy fraction for element $e, \delta E_{e}$ its potential energy density and $\alpha$ stands for an arbitrary weighting function. Here the notation $\mathbf{q}$ represents the vector of atomic positions while $\mathbf{u}$ represents the nodal displacements vector. In addition $\mathbf{q}_{\Omega^{A}}, \mathbf{q}_{\Omega^{R}}, \mathbf{u}_{\Omega^{F E}}$ and $\mathbf{u}_{\Omega^{R}}$ refer to spatial zones: they are used to indicate that a subset of the degrees of freedom (DOFs) is considered.

The two models are coupled in the bridging sub domain by constraining the DOFs in order to enforce the displacement continuity using a Lagrangian constraint formulation. The constraints in the bridging sub domain $\left(\Omega^{R}\right)$ hold on velocities and are expressed as:

$$
\forall i \in \Omega^{R} \quad \mathbf{g}_{\mathbf{i}}=\sum_{J} \varphi_{J}\left(\mathbf{X}_{\mathbf{i}}\right) \dot{\mathbf{u}}_{\mathbf{J}}-\dot{\mathbf{d}}_{\mathbf{i}}=0
$$

where $\varphi_{J}$ denotes the shape function associated to node $J$. For notation purposes, we consider indexes in upper case and in lower case to refer to finite-element quantities and atomic quantities respectively. If there are $L$ atoms in the coupling zone $\Omega^{R}$ then $L$ constraints are considered. The governing equations for the coupled multiscale domain are then formulated using the Lagrangian multiplier method. The Lagrange multipliers $\Lambda=\left(\lambda_{i}\right)_{i=1 . . L}$ are obtained by solving the linear system of equations

$$
\mathbf{H} \Lambda=\mathbf{g}^{\star}
$$

with $\mathbf{g}^{\star}$ the value of the constraint vector before correction and $\mathbf{H}$ is the $L \times L$ constraint matrix defined as

$$
H_{i j}=\sum_{J} \varphi_{J}\left(\mathbf{X}_{i}\right) \varphi_{J}\left(\mathbf{X}_{j}\right) \hat{M}_{J}^{-1}-\hat{m}_{i}^{-1} \delta_{i j}
$$

where $\hat{M}_{I}=\alpha\left(X_{I}\right) M_{I}$ and $\hat{m}_{i}=\left(1-\alpha\left(X_{i}\right)\right) m_{i}$, considering that $M_{I}$ is the lumped mass on node $I$ and $m_{i}$ is the mass of atom $i$. Finally, considering the modified forces $\hat{\mathbf{f}}_{i}$ and $\hat{\mathbf{f}}_{I}$ (due to the weighted Hamiltonian), the discrete governing equations within the two models are expressed as follows:

$$
\left\{\begin{array}{l}
\hat{M}_{I} \ddot{\mathbf{u}}_{I}=\hat{\mathbf{f}}_{I}-\sum_{k=1}^{L} \lambda_{k} \varphi_{I}\left(\mathbf{X}_{k}\right) \\
\hat{m}_{i} \ddot{\mathbf{d}}_{i}=\hat{\mathbf{f}}_{i}-\lambda_{i}
\end{array}\right.
$$

for which the integration in time is performed using the SHAKE algorithm [27]. Details of the derivation of the above equations are presented in $[19,20,23]$. We insist again on the fact that both FE and MD DOFs of the overlapping area are modified by the application of the constraint. This brings consequences in the finite temperature regime as discussed in the following subsection. 




Figure 1: Model used to test the Bridging Domain. The coupling area is presented in red, while the white upper zone is constituted of atoms and the white bottom zone is continuum only. The top blue layer is a region of fixed atoms that provides a boundary condition to the atomic model.

\subsection{Finite Temperature with the Bridging Domain}

Since we wish to investigate the influence of temperature on contact evolution, we seek to apply a finite temperature throughout the substrate body. To that purpose we use a thermostat in the atomic zone. The coupling scheme will change the temperature field as we demonstrate below. In essence the Bridging Domain can be considered as a projection that pushes gradually the atomic DOFs to the interpolated continuum fields. These are obviously smoother than the noisy atomic description of velocities. While we try to maintain constant temperature, the coupling scheme has a cooling effect on the coupled atoms.

Let us define the simplest patch test to evaluate the capacity of a coupling method at finite temperatures. This test considers a piece of material at uniform temperature and zero pressure. In such conditions, mechanical and thermodynamic equilibrium is initially set. Thus, for a robust coupling method, temperature should remain constant. Let us introduce the geometry defined in figure 1. The top part $\left(\Omega^{A}\right)$ is modelled with MD while the bottom part $\left(\Omega^{F E}\right)$ is driven by finite elements. The overlapping zone follows the Bridging Domain strategy. For the boundary conditions we maintain fixed the top layer of atoms (presented in blue), as well as the bottom free surface of the mesh. In the other two directions periodicity is imposed. The temperature is maintained to a constant value by means of a Berendsen [28] thermostat which includes a viscous force that is applied to thermostated atoms. This leads to a rescaling of atomic velocities by a parameter $\alpha$ following:

$$
\alpha=\sqrt{1+\frac{\triangle t}{\tau}\left(\frac{T_{0}}{T}-1\right)}
$$

where $\Delta t$ is the time step used, $\tau$ is a time parameter that sets the strength of the coupling with the heat bath, $T_{0}$ is the heat bath temperature and $T$ is the actual temperature of the domain. Such a thermostat is applied in the atomic zone $\Omega^{A} \backslash \Omega^{R}$ which excludes coupling and finite element 


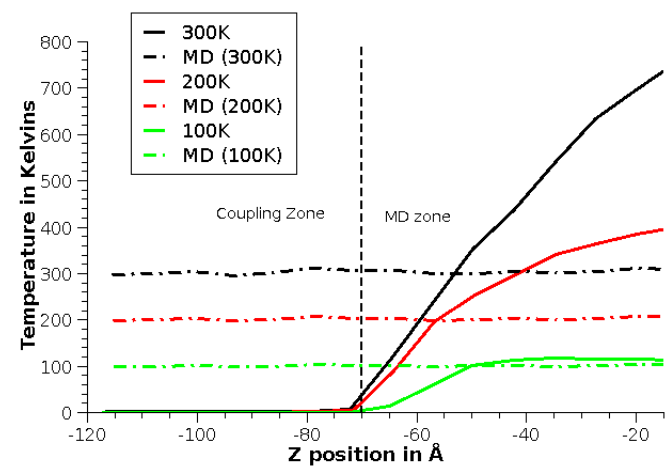

(a)

Figure 2: Temperature profile along the vertical direction (a). The decay of temperature is particularly evident. The temperatures were extracted along a vertical line as presented on the left image of (b). The kinetic energy removal happens very quickly. At time $t=0$ no projection is applied, and already at time $t=1 \mathrm{ps}$ the projection dropped almost all temperature in the coupling region as seen in (b). The temperature profiles issued from a full molecular dynamics simulation is presented for comparison purposes. The non-physical temperature gradient created is due to the projective nature of the Bridging Domain method. An important point to note is the impact on the non-coupled zone of the temperature profile which implies a strong modification of the dynamics in $\Omega^{A}$.

zones. Therefore, atoms in the coupling zone (colored in red) are driven by their proper dynamics and the coupling corrections.

The dynamic evolution of the model was computed with no load. Temperatures of 100, 200 and 300 Kelvins were considered. After a few time steps, the coupling has effectively changed the temperature profile as can be seen in figure 2. Also, the temperature profiles that are obtained by using only molecular dynamics with no coupling were extracted and are presented in this figure. Again, the temperature through the bridging zone cannot be constant since the coupling algorithm projects the noisy velocities of single atoms to the much smoother continuum velocity field. The difference in scale from atomic to the defined mesh leads to an impossibility of representing very high frequencies. Therefore, as heat within MD is represented by atomic lattice vibrations, the projection results in a temperature drop in the bridging zone. We note here that this has also an effect on the atoms in $\Omega^{A}$ since the Berendsen thermostat is maintained globally. This explains why, in a region, the temperature is above the thermostated temperature (see figure 2). Clearly, our coupling (and projection) strategy is not suitable for a finite temperature problem since it impacts temperature also in the uncoupled zone.

This temperature change induces yet another side effect. Indeed, the introductory part presented that the volume of the copper crystal was set accordingly to maintain zero pressure and constant temperature. Since the projection modifies the kinetic energy of the coupled atoms, these want to shrink their attributed volume in order to go back to ground state. Ultimately, this temperature decrease manifests mechanically as an internal spurious displacement along the negative temperature gradient in the coupled area. Arguably, the observed displacements are small $(\sim 0.8 \AA$ at $500 \mathrm{~K})$ and may be considered as a small side effect compared to the temperature gradient.

Despite our best efforts, we could not find an easy scheme to compensate for this temperature gradient and the shrinkage sustained by the coupled atoms. Since the coupling damps quite strongly kinetic energy it makes any thermostat in $\Omega^{R}$ unusable (and unstable) to fix this temperature decrease. Concerning the internal displacement, the use of a force in a single direction 




(a)

(b)

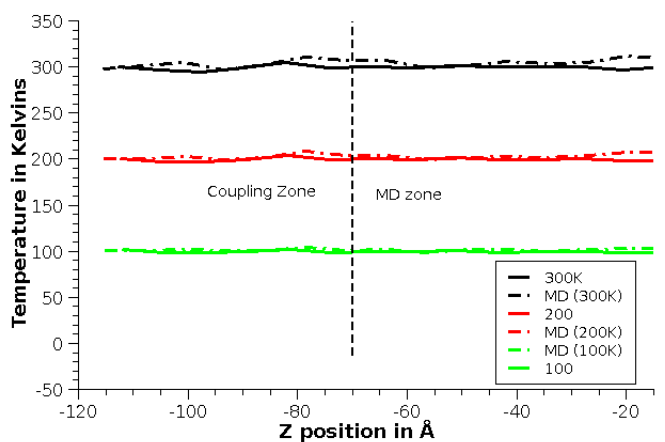

Figure 3: On subfigure (a) we present the proposed coupled domain used with finite temperature. Besides the atomic zone $\left(\Omega^{A}\right)$ and the continuum zone $\left(\Omega^{F E}\right)$, two zones are defined where coupling is done separately. In $\Omega^{1}$ mesh nodes are constrained to atomic mean displacement and in $\Omega^{2}$ atomic displacements are interpolated from the mesh to provide a moving boundary condition to $\Omega^{A}$. Subfigure (b) shows the temperature profiles obtained with the method proposed in section 3.3 .

cannot compensate a volumetric change. A patch to these problems would require changing the inter-atomic potential, which is technically complex. Instead, we changed the coupling method, so that no temperature cooling would take place. This is the purpose of the next section.

\subsection{Multiscale coupling at finite temperature}

In order to achieve a reasonable coupling that does not lead to an artificial cooling of the system, the previously presented set of equations must be changed. Indeed, we need to remove any projection that modifies the atomic field and thus would damp a part of the kinetic energy. We will separate the coupling in two zones $\Omega^{1}$ and $\Omega^{2}$ as depicted in figure 3 . In the first one the atomic DOFs will not be modified whereas the continuum DOFs will be constrained to match the atomic solution. Thus the kinetic energy of these atoms is left untouched preventing the previously mentioned side effects and providing a mechanism for passing information from MD to FE. In $\Omega^{2}$, the reverse operation is performed by constraining atoms to the continuum fields by means of a simple interpolation. One inconvenient is that no wave reflection treatment is introduced here but The following procedure is thought only for constant finite temperatures.

In $\Omega^{1}$ we constrain the mesh nodes to atomic displacements. Since mesh nodes in the bridging zone are now fictive and purely driven from $\mathrm{MD}$, let us consider the following constraints:

$$
\mathbf{g}_{\mathbf{i}}=\sum_{J} \varphi_{J}\left(\mathbf{X}_{\mathbf{i}}\right) \mathbf{u}_{\mathbf{J}}-\mathbf{d}_{\mathbf{i}}
$$

where mesh nodes are to be corrected and the atomic velocities and positions are not modified. 
In $\Omega^{1}$ the governing equation for continuum DOFs is:

$$
M_{I} \ddot{\mathbf{u}}_{I}=\mathbf{f}_{I}-\sum_{k=1}^{L} \lambda_{k} \varphi_{I}\left(\mathbf{X}_{\mathbf{k}}\right)
$$

The SHAKE [27] integration scheme provides:

$$
\left\{\begin{array}{l}
\dot{\mathbf{u}}_{I}^{n+1 / 2}=\dot{\mathbf{u}}_{I}^{n}+\frac{\Delta t}{2 M_{I}} \mathbf{f}_{I}^{n}-\frac{\Delta t}{2 M_{I}} \sum_{k=1}^{L} \lambda_{k} \varphi_{I}\left(\mathbf{X}_{\mathbf{k}}\right) \\
\mathbf{u}_{I}^{n+1}=\mathbf{u}_{I}^{n+1}+\Delta t \dot{\mathbf{u}}_{I}^{n+1 / 2} \\
\text { evaluation of } \mathbf{f}_{I}^{\mathbf{n}+1} \\
\dot{\mathbf{u}}_{I}^{n+1}=\dot{\mathbf{u}}_{I}^{n+1 / 2}+\frac{\Delta t}{2 M_{I}} \mathbf{f}_{I}^{n+1}
\end{array}\right.
$$

with the requirement that the constraint function should be respected, we combine 3.10 and 3.12 to obtain:

$$
\sum_{J} \varphi_{J}\left(\mathbf{X}_{\mathbf{i}}\right) \mathbf{u}_{\mathbf{J}}^{\star}-\mathbf{d}_{\mathbf{i}}=\sum_{k=1}^{L}\left(\sum_{J} \frac{\varphi_{J}\left(\mathbf{X}_{\mathbf{i}}\right) \varphi_{I}\left(\mathbf{X}_{\mathbf{k}}\right)}{M_{J}}\right) \lambda_{k}
$$

Where $\mathbf{u}_{J}^{\star n+1}=\mathbf{u}_{I}^{n}+\dot{\mathbf{u}}_{I}^{n}+\frac{\Delta t^{2}}{2 M_{I}} \mathbf{f}_{I}^{n}$. It also means that $\star$ superscript denotes displacement values before the application of the constraint. Thus a new constraint matrix $\mathbf{H}$ is defined so that:

$$
H_{i j}=\sum_{J} \frac{\varphi_{J}\left(\mathbf{X}_{\mathbf{i}}\right) \varphi_{I}\left(\mathbf{X}_{\mathbf{j}}\right)}{M_{J}}
$$

It leads to a comparable linear system $\mathbf{H} \lambda=\mathbf{g}^{\star}$ to solve in order to compute the multipliers. Once the Lagrange multipliers are obtained, we can constrain the nodal displacements to a coherent state with the atomic displacements. However the formulation also needs a correction of the velocities. Finally, the following algorithm is to be used:

$$
\begin{aligned}
& \text { (1) } \dot{\mathbf{u}}_{I}^{\star}=\dot{\mathbf{u}}_{I}^{n}+\frac{\Delta t}{2 M_{I}} \mathbf{f}_{I}^{n} \\
& \text { (2) } \mathbf{u}_{I}^{\star}=\mathbf{u}_{I}^{n}+\Delta t \dot{\mathbf{u}}_{I}^{\star} \\
& \text { (3) } \mathbf{g}_{\mathbf{i}}^{\star}=\sum_{J} \varphi_{J}\left(\mathbf{X}_{\mathbf{i}}\right) \mathbf{u}_{\mathbf{J}}^{\star}-\mathbf{d}_{\mathbf{i}}^{\mathbf{n + 1}} \\
& \text { (4) } \lambda_{\mathbf{i}}=H_{i k}^{-1} \mathbf{g}_{i}^{\star} \\
& \text { (5) } \dot{\mathbf{u}}_{I}^{n+1 / 2}=\dot{\mathbf{u}}_{I}^{\star}-\frac{\Delta t}{2 M_{I}} \sum_{k=1}^{L} \lambda_{k} \varphi_{I}\left(\mathbf{X}_{\mathbf{k}}\right) \\
& \text { (6) } \mathbf{u}_{I}^{n+1}=\mathbf{u}_{I}^{\star}-\frac{\Delta I^{2}}{2 M_{I}} \sum_{k=1}^{L} \lambda_{k} \varphi_{I}\left(\mathbf{X}_{\mathbf{k}}\right) \\
& \text { (7) } \text { evaluation of } \mathbf{f}_{I}^{\mathbf{n}+1} \\
& \text { (8) } \dot{\mathbf{u}}_{I}^{n+1}=\dot{\mathbf{u}}_{I}^{n+1 / 2}+\frac{\Delta t}{2 M_{I}} \mathbf{f}_{I}^{n+1} \\
& \text { (9) Go back to (1) }
\end{aligned}
$$

Clearly this creates a one way coupling process from MD to FE. The reverse exchange of information is then processed in $\Omega^{2}$ by providing a moving boundary condition to the atomic model. The temperature of boundary atoms is now related to the vibration modes of the finite element part. Since the velocity of mesh nodes are smaller, because of the change of scale, the imposed velocity onto the atoms is almost zero. Nevertheless no thermal energy flux is occurring in that area, as revealed in figure 3 which shows a constant temperature profile.

This method was used with the previously defined patch test with success. We also loaded the top surface and verified that the final static state was coherent with continuum theory. Nevertheless this method can probably not be applicable in NVE (number of particles, volume and energy 
remain constant) ensemble at very low temperatures since the amount of kinetic energy trapped by reflections at the interface would modify the dynamics of the MD region. But projection methods are not applicable here as well due to thermal expansion.

Our proposed coupling scheme shares common features with seamless methods [17]. One may therefore question the utility of bridging zone at all when no wave reflection treatment is performed. Indeed, CADD method has been used at finite temperature with nodal time averaging to transfer mechanical fields at thermodynamic equilibrium [17]. Here, the same goal is achieved, but the overlap region opens perspectives for computation of local temperatures and heat fluxes which seems very difficult in the context of an edge-to-edge coupling formulation. Another advantage of overlapping methods stands in the reduction of the number of degree of freedom since, in opposition to seamless methods, the finite element mesh does not need to be refined down to the atomic scale. Our method is thought to be a starting point for an energy balance equation that would lead to proper treatment of energy fluxes.

\section{Results}

This section presents the obtained results concerning a normal loading of rough (self-affine fractal) surfaces. Our setting considers the loading of a flat surface (moving downwards) onto a deformable rough surface which is maintained at a desired temperature. To construct such a surface we first consider a pristine piece of crystalline copper in which we cut a fractal surface. The description of the fractal surface is given by a Voss $[29,30]$ algorithm. Also we took into account the thermal expansion described in section 2. We prepared two 3D geometries with different surface roughness. As illustrated in figure 4, one surface was parameterized to a Hurst exponent $H=0.7$ and root mean square of heights $\Delta^{r m s}=10 a$ while a flatter one was set to have $H=0.8$ and $\Delta^{r m s}=5 a$. In both cases, the section is $L^{2}$ with $L=32 a$, which is small enough to allow fast computations on a parallel computing machine while having around 100000 atoms in the atomic zone. The deepness of the atomic domain is also taken as $32 a$ but since some layers where removed at the free surface the exact global height is effectively a little reduced. Nevertheless, the coupling allows to have an additional elastic zone of $64 a$ being 128 additional atomic layers. The mesh is made of $10 \times 10 \times 20$ elements. While the inter-atomic potential used for the substrate is the EAM described previously, the interactions between atoms from the rigid plate and substrate have been modified: a Lennard Jones potential with a cutoff radius of $a \sqrt{2} / 2$ (first neighbors distance) is used in order to discard the adhesive forces (removing the adhesive forces when using EAM is a complicated task since the electronic contribution is not a pair-wise term).

Before loading the rigid plane the substrate is allowed to equilibrate to a required temperature, which modifies the surface as will be presented later. Again, the constant temperature was constrained using a Berendsen thermostat [28]. The thermostat was applied on the molecular part since the continuum zone only handles mechanics.

After equilibration, we allow the top flat rigid surface to descend downwards and create contact in reaction to an applied initial pressure of $0.1 \mathrm{GPa}$. The pressure is regularly incremented by 0.1 GPa every $10 p s$. Thus the loaded surface has $10 p s$ to equilibrate, which establishes a quasi-static loading of our substrate.

The simulation was run on a parallel cluster, with one processor dedicated to finite element elasticity, while 32 processors were occupied in the time integration of molecular dynamics. 


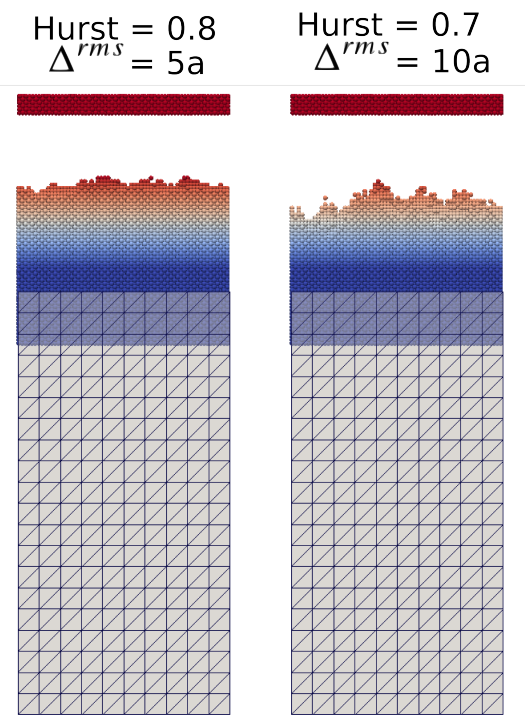

Figure 4: The surface profiles obtained with a Voss [29, 30] algorithm are presented. Underneath, the substrate is composed of copper atoms. The flat body above the substrates is the rigid surface to be pressed against the rough surfaces. The left surface is flatter than the one on the right.

We used LAMMPS ${ }^{1}$ for molecular dynamics and a in-house code for finite elements, while LibMultiScale [31] was carrying the coupling process.

\subsection{Measurement method for contact area and load}

As our objective is to follow the evolution of the contact area with load, it is necessary to monitor precisely the applied pressure. We impose a constant pressure on the rigid body following the same strategy depicted in [32]. We set the center of mass of the top body to move according to a unique force. This force is computed with:

$$
F=\sum_{a t} f^{a t}-P \cdot L^{2}
$$

where $P$ is the desired pressure, and $f^{a t}$ is the initial per atom force issued from standard molecular dynamics. We then "distribute" such a force over all the atoms so that the resulting atomic acceleration becomes uniform and follows:

$$
\forall i \in \Omega^{\text {rigid }}, \quad a_{i}=\frac{\sum_{a t} f^{a t}-P \cdot L^{2}}{m_{r}}
$$

with $\Omega^{\text {rigid }}$ the domain of the rigid plate and $m_{r}$ the mass assigned to each atoms in $\Omega^{\text {rigid }}$. We reduced arbitrarily the mass of these atoms so that the contacting force is transmitted with reduced inertial effects. We used $m_{r}=\epsilon m_{c u}$ with $m_{c u}$ being the mass of a single copper atom. Having $\epsilon<<1$ the rigid body is light enough to move freely, therefore enforcing an almost constant pressure at the contacting asperities.

\footnotetext{
${ }^{1}$ http://lammps.sandia.gov/
} 


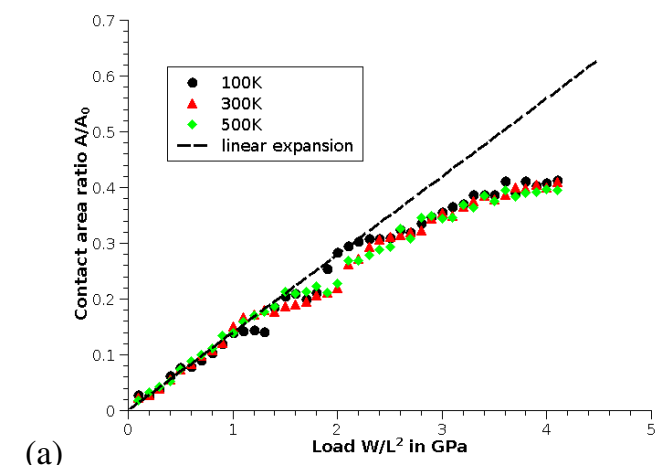

(b)



Figure 5: Contact area ratio $A / L^{2}$ with respect to the applied load expressed in GPa. The left graph is obtained from the $H=0.8$ (a) surface while on the right is from the $H=0.7$ (b) surface.

Concerning the contact area ratio, it is to be understood that we have to manage a data structure to represent atoms (or points). A contacting point is then defined by the position of a substrate atom that is close enough (closer than $a \sqrt{2} / 2$ ) to an atom from the upper body. In addition, contacting particles should be accounted in surface area units implying a projection on the horizontal plane.

\subsection{Contact area ratio versus load}

We loaded the two surfaces from $0.1 \mathrm{GPa}$ to $4 \mathrm{GPa}$ and measured contact area. The results are presented in figure 5 for the two surfaces separately. The first observation is that, in accordance to continuum theories, we observe a linear regime for small loads. The linearity ends for both surfaces at around 1.5 GPa corresponding to $20 \%$ of contact. Beyond the threshold that defines linear regime, important plastic events occur at the surface. Two kind of mechanisms, which will be described in section 4.3, lead to hardening of the material at the surface. This is characterized by the change of slope, in the contact area ratio versus load of figure 5 and 6 . The other important remark is that the temperature does not seem to change substantially the observed measures. This is surprising since the elastic constants are temperature dependent. Still, it does not mean that temperature has no impact on the mechanics as will be shown below.

Figure 6 contrasts the contact area ratio obtained with the two different surfaces. It appears that the linear regimes are almost comparable. However the flatter surface presents a greater hardening. This can be explained by the extent of plastic activity in the bulk. Indeed, the non reversible phenomena that can occur in the substrate have been identified to be of two kinds at this scale [10]: reshuffling of atoms and dislocation emissions. These two mechanisms both exert influence on the evolution of the contact area with load. Dislocation emission is dominant for the flattest surfaces $(H=0.8)$ and generates the strongest hardening. When surfaces become rougher $(H=0.7)$, another important mechanism emerges, atom reshuffling, that takes the form of atoms being pushed around at the surface. In view of figure 6, atom shuffling brings local hardening that resolves by itself after further loading and reshuffling (stepped behavior in figure $5 b)$. The next subsection will present how we identified plastic events and their energy activation in relation to temperature. 


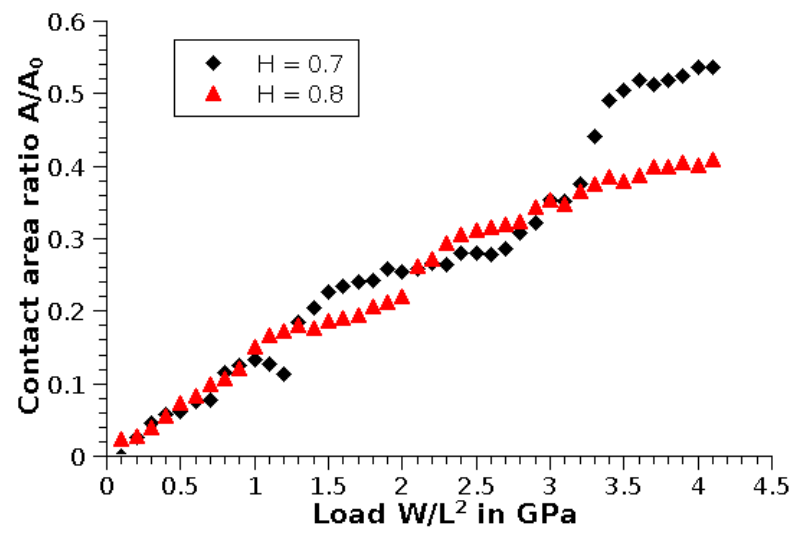

Figure 6: Contact area ratio with respect to load at 300 Kelvins for the two studied surfaces.
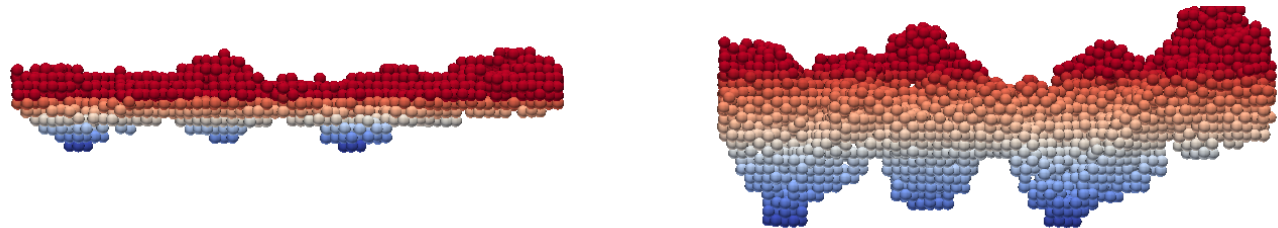

Figure 7: Atoms that changed neighbors after relaxation was performed. The left is for $H=0.8$ while the right is for $H=0.7$. Note that the rougher surface goes through more intense reshuffling.

\subsection{Plastic activity}

A plastic event, be it a dislocation or a non reversible motion of atom can be characterized at the atomic scale by a simple criterion. Indeed, while during an elastic deformation a given atom is displaced with all its neighbors, when a dislocation is created a slip system is activated. Along this slip plane, atoms have changed neighbors. The same happens for any non reversible process. Therefore as in [10] we track the atoms that changed neighbors during the simulation.

Figures 7 and 8 present the atoms selected based on such a criterion. Figure 7 shows these atoms just after surface relaxation. Since all the free surface appears in the figure, we can state that during the initial relaxation these surfaces were subject to intense reshuffling. Then, figure 8 presents the simulation at a later time, for an applied load of $1 G P a$. The $H=0.8$ surface generated a dislocation into the bulk part whereas the rougher surface only reshuffled surface atoms. This can also be seen on figure 9 in which the plastic events occurring after the initial relaxation are counted.

In the case of the flatter surface, the plastic count only increases slowly until around 800MPa. Then the dislocation presented on figure 8 is created and is seen by the important increase in the plastic counter. Also it is well known that the temperature impacts the activation energy of dislocations and this is observable here. Indeed, the plastic counter jump appears later for lower temperatures (figure 9). In contrast, the rougher surface (curve on the right) shows more gradual plastic activity, with slightly higher slope, but with no dislocation emission. Furthermore, it appears that the plastic reshuffling is not temperature dependent (at least not as clearly as the 



Figure 8: Atoms that changed neighbors when the applied load is $1 G P a$. The left is the for $H=0.8$ while the right is for $H=0.7$. Note the dislocation emission on the left image.
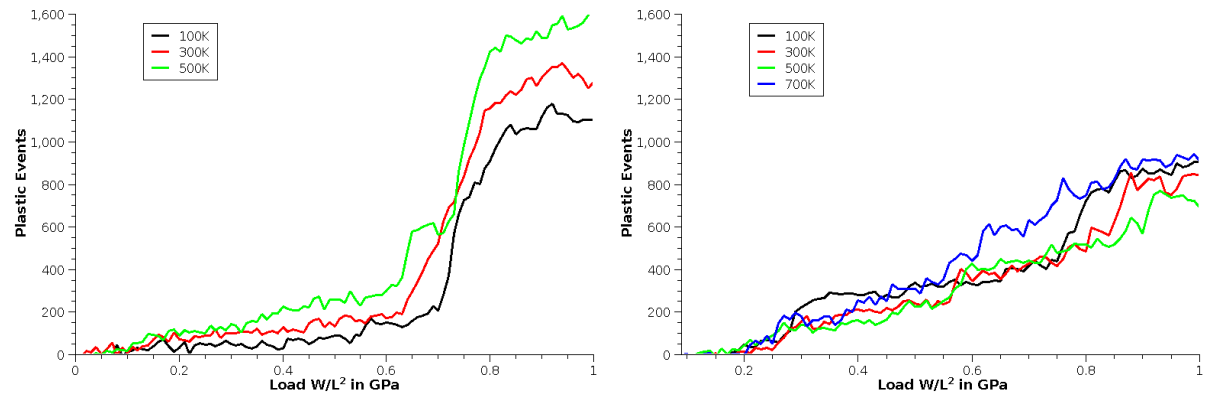

Figure 9: Plastic event counter with respect to applied load in GPa. The results for the flatter $(H=0.8)$ and rougher surfaces $(H=0.7)$ at $300 \mathrm{~K}$ are presented on the left and right figures respectively. 


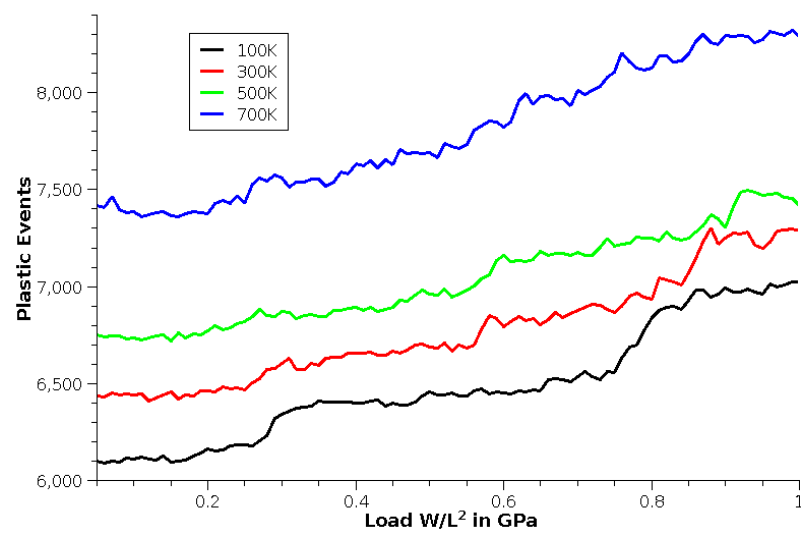

Figure 10: Absolute plastic events detected for the surface with $H=0.7$ at various temperatures.

dislocation activation threshold is).

Overall, it seems that in our calculations the reshuffling of the atoms at the free surface is much more predominant than what is observed in 2D [10]. We believe that this is due to the three-dimensional geometry used here which allows a rearrangement of the atoms in all directions making this phenomenon less energy consuming. This is also believed to be a possible explanation for the non dependency of the contact area with respect to the temperature even though elastic constant are temperature dependent.

However, "plastic" relaxation of surfaces is clearly temperature dependent. Figure 10 shows the absolute plastic counter for the rougher surface that accounts for atoms that lost their neighbors during relaxation. The temperature plays an important role in the modification of the topology: the warmer the surface is, the less stable it is. The destruction of the roughness is thus effective with no load. This means simply that the surface fractal characteristics that were used to build our model were modified during that initial stage. Figure 11 shows the increment of plastic activity during relaxation with respect to temperature (the plastic level at $100 \mathrm{~K}$ was taken as a reference). The rougher surface is much less stable than the flatter one as stated by the progression of the data points of figure 11 .

\section{Conclusion}

In this paper, a methodology to study nanoscale normal contact of a rough surface at finite temperature has been introduced. The main simulation tool is Molecular Dynamics but a coupling algorithm was introduced in order to lower the computational cost. The actual gain of the approach allowed to reduce by a factor two the computation time. This gain is expected to increase with larger models.

We showed that the Bridging Domain method (and more generally projective approaches) cannot handle finite-temperature coupling since the development of strong thermal gradients and related thermal expansion and volume forces are extremely difficult to correct. We then proposed a coupling strategy that could successfully prevent the spurious effects exhibited by the Bridging Domain method. The advantages of our approach are that the coupling zone needs a priori no 


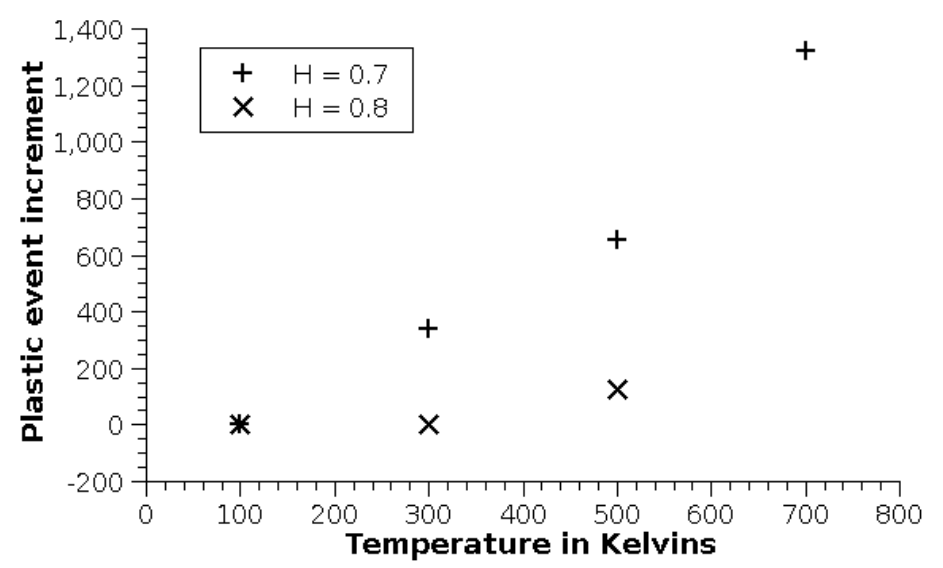

Figure 11: Plastic event after initial relaxation with respect to the temperature for the two studied surfaces.

geometrical pre-requisites and that it can be much smaller than its bridging-zone counterpart. However, the catch is that the method looses the ability to absorb high frequencies at 0 Kelvin.

This approach was tested with the normal loading of two rough surfaces. We measured the real contact area as a function of the applied pressure. In accordance with experimental and theoretical evidence, we observed a linear dependence of area on load, and this even if plastic deformation mechanisms were dominating. Surprisingly, we could not observe a dependence of the slope on temperature. As the elastic constants decrease with temperature, it was expected that the slope would drop as well. The mechanisms for this are still unclear. A possible explanation is that plasticity is the dominant effect at this scale. Indeed, we have shown evidence of intense plastic activity and this in two forms, dislocation emission and reshuffling of the atoms at the surface. For a large range of loadings reshuffling is the main mechanism behind plasticity. This mechanism appears to be particularly important in our 3D study, hinting at a clear difference with 2D studies in which surface atom motion can occur only in two directions. Our results indicate that atomic shuffling initiated during contact is not temperature sensitive. Atomic shuffling mechanisms seem also to take more importance for rougher surfaces.

Yet another possible explanation for the lack of temperature dependence of the area versus load curve is our system size. One could argue that the section used does not contain enough atoms to form a substantial statistical data set. The noise generated might prevent us to extract contact measures with precision. In future work, we will take full advantage of the multiscale methodology and study much larger sections. Also, the relaxation of atomic surfaces at finite temperatures has opened the question of the definition of atomic roughness. Indeed, we may put in question the notion that surfaces show drastic change of slopes at the atomic scale. Studying larger sections would allow us to represent fractal surfaces at the scale of 'microscopic' asperities while considering smooth surfaces with perhaps a few atomic steps at the finer scale. Finally, our future studies will include the development of coupling methods that can handle thermal fluxes as these may become dominant in sliding contact applications. 


\section{Acknowledgments}

This material is based on the work supported by the Swiss National Foundation under Grant no 200021_122046/1 and the European Research Council Starting Grant no 240332. The authors would like to acknowledge useful discussions with Dr Peter Spijker.

\section{References}

[1] K.L. Johnson. Contact mechanics. Cambridge, New York, 1985.

[2] A.W. Bush, R.D. Gibson, and T.R. Thomas. Elastic contact of a rough surface. Wear, 35(1):87-111, 1975. ISSN 0043-1648.

[3] A.A. Polycarpou and I Etsion. Analytical approximations in modeling contacting rough surfaces. Journal of Tribology-Transactions of the ASME, 121(2):234-239, Apr 1999. ISSN 0742-4787. ASME/STLE Tribology Conference, Toronto, Canada, Oct 25-29, 1998.

[4] B.N.J. Persson. Elastoplastic contact between randomly rough surfaces. Physical Review Letters, 87(11), Sep 10 2001. ISSN 0031-9007. doi: 10.1103/PhysRevLett.87.116101.

[5] S Hyun, L Pei, J.F. Molinari, and M.O. Robbins. Finite-element analysis of contact between elastic self-affine surfaces. Physical Review E, 70(2, Part 2), Aug 2004. ISSN 1063-651X. doi: 10.1103/PhysRevE.70.026117.

[6] L Pei, S Hyun, J.F. Molinari, and M.O. Robbins. Finite element modeling of elasto-plastic contact between rough surfaces. Journal of the mechanics and physics of solids, 53(11):2385-2409, Nov 2005. ISSN 0022-5096. doi: 10.1016/j.jmps.2005.06.008.

[7] C Campana and M.H. Mueser. Contact mechanics of real vs. randomly rough surfaces: A Green's function molecular dynamics study. EPL, 77(3), 2007. ISSN 0295-5075. doi: 10.1209/0295-5075/77/38005.

[8] S Hyun and M.O. Robbins. Elastic contact between rough surfaces: Effect of roughness at large and small wavelengths. Tribology International, 40(10-12):1413-1422, Oct-Dec 2007. ISSN 0301-679X. doi: 10.1016/j.triboint.2007.02.003. 33rd Leeds-Lyon Symposium on Tribology, Leeds, England, Sep 12-15, 2006.

[9] M Ciavarella, C Murolo, and G Demelio. On the elastic contact of rough surfaces: Numerical experiments and comparisons with recent theories. Wear, 261(10):1102-1113, Nov 30 2006. ISSN 0043-1648. doi: 10.1016/j.wear.2006.02.001

[10] B.Q. Luan and M.O. Robbins. Hybrid Atomistic/Continuum Study of Contact and Friction Between Rough Solids. Tribology Letters, 36(1):1-16, Oct 2009. ISSN 1023-8883. doi: 10.1007/s11249-009-9453-3.

[11] B.Q. Luan, S Hyun, J.F. Molinari, N Bernstein, and M.O. Robbins. Multiscale modeling of two-dimensional contacts. Physical Review E, 74(4, Part 2), Oct 2006. ISSN 1539-3755. doi: 10.1103/PhysRevE.74.046710.

[12] F.P. Bowden and D. Tabor. Lubrication of Solids. Oxford University Press, 1950.

[13] P. Wriggers and C. Miehe. Contact constraints within coupled thermomechanical analysisA finite element model. Computer Methods in Applied Mechanics and Engineering, 113(34):301-319, March 1994. ISSN 0045-7825. doi: 10.1016/0045-7825(94)90051-5. URL http://www. sciencedirect.com/science/article/B6V29-47XF93B-9K/2/768e49941bf 433222d466063511030f7.

[14] J.Q. Broughton, F.F. Abraham, N Bernstein, and E Kaxiras. Concurrent coupling of length scales: Methodology and application. Physical Review B, 60(4):2391-2403, Jul 15 1999. ISSN 0163-1829.

[15] S Kohlhoff, P Gumbsch, and H.F. Fischmeister. Crack-propagation in bcc crystals studied with a combined finiteelement and atomistic model. Philosophical Magazine A-Physics of condensed matter structure defects and mechanical properties, 64(4):851-878, Oct 1991. ISSN 0141-8610.

[16] R Miller, E.B. Tadmor, R Phillips, and M Ortiz. Quasicontinuum simulation of fracture at the atomic scale. Modelling and Simulation in Materials Science and Engineering, 6(5):607-638, Sep 1998. ISSN 0965-0393. International Conference on Computational Physics, Santa Cruz, California, AUG 25-28, 1997.

[17] S Qu, V Shastry, W.A. Curtin, and R.E. Miller. A finite-temperature dynamic coupled atomistic/discrete dislocation method. Modelling and Simulation in Materials Science and Engineering, 13(7):1101-1118, Oct 2005. ISSN 0965-0393. doi: 10.1088/0965-0393/13/7/007.

[18] J Marian, G Venturini, B.L. Hansen, J Knap, M Ortiz, and G.H. Campbell. Finite-temperature extension of the quasicontinuum method using Langevin dynamics: entropy losses and analysis of errors. Modelling and Simulation in Materials Science and Engineering, 18(1), Jan 2010. ISSN 0965-0393. doi: 10.1088/0965-0393/18/1/015003.

[19] S.P. Xiao and T Belytschko. A bridging domain method for coupling continua with molecular dynamics. Computer Methods in Applied Mechanics and Engineering, 193(17-20):1645-1669, May 2004. ISSN 0045-7825. doi: 10.1016/j.cma.2003.12.053.

[20] T Belytschko and S.P. Xiao. Coupling methods for continuum model with molecular model. International Journal for Multiscale Computational Engineering, 1(1):115-126, 2003 
[21] High Performance Multiscale Simulation for Crack Propagation, Aug 2006. HPSEC'2006.

[22] G Anciaux. Simulation multi-échelles des solides par une approche couplée dynamique moléculaire / éléments finis. De la modélisation à la simulation haute performances. $\mathrm{PhD}$ thesis, Université Bordeaux 1, Jul 2007.

[23] G Anciaux, O Coulaud, J Roman, and G Zerah. Ghost force reduction and spectral analysis of the 1d bridging method. INRIA report, HAL, 2008

[24] M.S. Daw and M.I. Baskes. Embedded atom method: Derivation and application to impurities, surfaces and other defects in metals. Physical Review B, 29:6443-6543, 1984.

[25] Y Mishin, M Mehl, D Papaconstantopoulos, A Voter, and J Kress. Structural stability and lattice defects in copper: $\mathrm{Ab}$ initio, tight-binding, and embedded-atom calculations. Physical Review B, 63(22), 2001. ISSN 0163-1829. doi: 10.1103/PhysRevB.63.224106.

[26] S Kazanc, Y.Ö. Çiftci, K Çolakoglu, and S Ozgen. Temperature and pressure dependence of the some elastic and lattice dynamical properties of copper: a molecular dynamics study. Physica B: Condensed Matter, 381(1-2): 96-102, May 2006. ISSN 0921-4526. doi: 10.1016/j.physb.2005.12.259.

[27] J.P. Ryckaert, G Ciccotti, and H.J.C. Berendsen. Numerical integration of cartesian equations of motion of a system with constraints - Molecular-Dynamics of N-Alkanes. Journal of Computational Physics, 23(3):327-341, 1977 ISSN 0021-9991.

[28] H.J.C. Berendsen, J.P.M. Postma, W.F. van Gunsteren, A DiNola, and J.R. Haak. Molecular dynamics with coupling to an external bath. The Journal of Chemical Physics, 81(8):3684-3690, October 1984. doi: 10.1063/1.448118. URL http://link. aip.org/link/?JCP/81/3684/1.

[29] H.O. Peitgen and D Saupe. The science of Fractal image. Springer-Verlag New York, 1988.

[30] R.F. Voss. Fundamental algoritmhs in computer graphics. Springer-Verlag Berlin, 1985.

[31] G Anciaux. Libmultiscale framework based on bridging domain method. http://libmultiscale.gforge.inria.fr/, 2007. URL http://libmultiscale.gforge.inria.fr/.

[32] G Anciaux and J.F. Molinari. Sliding of rough surfaces and energy dissipation with a 3d multiscale approach International Journal for Numerical Methods in Engineering, Early view(DOI:10.1002/nme.2845), 2010. 